\title{
Novel Distance Estimation Algorithm of Two-hop Neighbor in Wireless Sensor Networks
}

\author{
Sungchang Choi, and Joongsoo Ma \\ Department of Electrical Engineering, Korea Advanced Institute of Science and Technology, Korea \\ \{nangchoi, jsma\}@kaist.ac.kr
}

\begin{abstract}
In case of wireless networks which does not contain a localization system, Euclidean distance between two nodes those are out of carrier sensing range each other cannot be measured with ranging techniques such as RSSI, ToA, and TDoA since they are not able to communicate directly. To deal with this issue, range-free schemes like a hop-count based approach and a connectivity based approach were used. These methods, however, are decreased in accuracy at low node density networks, so it is hard to apply to other wireless network except the densely deployed sensor networks. In this paper, we present a novel distance estimation method. When two nodes are in 2-hop neighbor relation, our method can estimate a distance using ranging information of their overlapped neighbors and connectivity information. Simulation results show that the proposed method outperforms hop count based and connectivity based distance estimation methods.
\end{abstract}

Keywords-Wireless sensor networks, distance estimation, interference range.

\section{INTRODUCTION AND RELATED WORK}

In wireless networks, distance estimation is a principal technology since it is used to routing, localization, interference estimation, etc. So more accurate distance estimation brings more accurate positioning, efficient routing, and exact hidden node estimation.

Distance estimation methods can be divided into the rangebased method and the range-free method. Typical range-free schemes are hop-count based (HCB) approach and connectivity based approach. HCB approach [1] is a simple distance estimation technique which calculate the distance by multiplying the smallest hop count number between two nodes by average one hop distance. This method has relatively low accuracy at low density but in high node density, it performs well so it is proper to sensor networks. Connectivity based approach assumes that nodes are uniformly distributed. If there are two nodes that we want to know the distance of them, connectivity based approach [2] exploits the number of shared neighbors and total neighbors to calculate an overlapped area. Then geometric formulations are used to estimate the distance. These two approaches have large estimation error in low node density environment.

The range-based methods directly measure the distance between two nodes by using received signal strength (RSS), time of arrival (ToA), time difference of arrival (TDoA). These methods are not able to measure the distance if receiver is out of transmission range of sender since receiver may not detect the signal. To solve the problems, some studies are proposed. First remedy is exploiting localization to construct a relative coordinate system [3]. If each node has more than 3 nodes, it can make a arbitrary coordinate system and is able to define its relative position. Select one criterion of the coordinate system above various relative coordinate systems. Then, it is possible to unify the different coordinate systems of each node. So the positions of all nodes in the networks are redefined for the certain coordinate system. After then the distance calculation between two nodes is simple. Drawbacks of this approach are making relative coordinate systems is complicated and accumulated errors from distance estimation are multiplied when unifying coordinates. As a result, its estimation performance is poor. Another solution is the transmission power increase of sender node. Transmission power, however, increase will influence other links or networks. Moreover each country makes the maximum transmission power of wireless communications into a law, so enhancing the transmission power is restrictive. Another study [5]is a reducing the receiver sensitivity. It makes nodes have too large carrier sensing range and will reduce the network throughput significantly. The major disadvantage of [5] is a reduced effective transmission range.

In this paper, we propose a novel algorithm that estimates the two-hop neighbor distance with range and range-free information synthetically. Distance estimation is possible with the one of two schemes, range-based or range-free. However, range-based method is not able to measure the distance directly if target nodes are out of transmission range, and range-free scheme has worse performance in low node density networks. Exploiting two type of information concurrently makes us to achieve more precise distance estimation by making up for their shortcomings. Although we select RSS for ranging-based technique in this paper, any of techniques is possible. e.g., ToA, TDoA, etc. Simulation results show our method reduces estimation error more than $18 \%$ comparing to connectivity based algorithm and hop-count based algorithm.

The remainder of the paper is organized as follows. Section II presents proposed algorithm. After evaluating the performance via simulations in Section III, and Section IV concludes the papers.

\section{PRoposed Scheme}

Finding the node-to-node distance is the first step for most of the localization algorithms. Thus, many studies have been proposed for 1-hop distance estimation, and we may adopt suitable distance estimation or measurement techniques. However, the research that estimates the distance with more than 2-hop neighbor nodes is rare. We suggest a new 2hop distance estimation algorithm. In this work, we combine ranging-based approach with connectivity based method to 


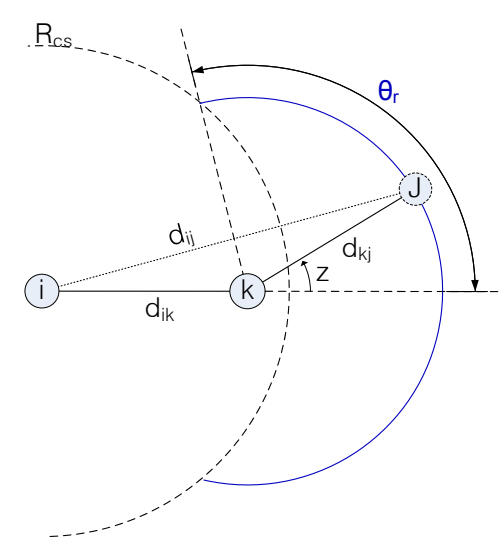

Fig. 1. Illustration of $\theta_{r}$ and available section of $z$

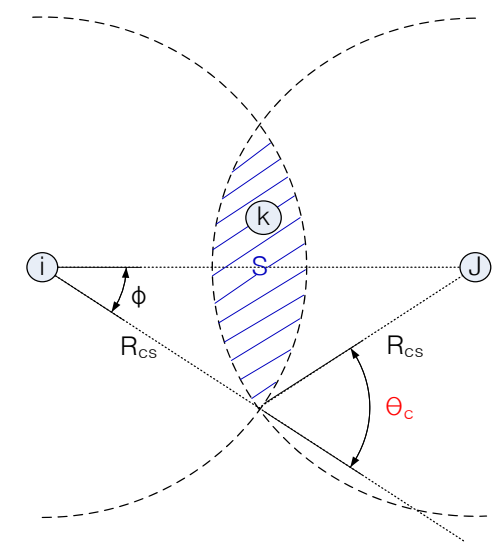

Fig. 2. Illustration of $\theta_{c}$ and intersection area, $S$

complement existing distance estimation studies that cannot estimate distance of more than 2-hop neighbors. In Figure $1, d_{i j}$ means Euclidean distance between node $i$ and $j$, and $R_{C S}$ represents the carrier sensing range. In this paper, we assume that all nodes have same carrier sensing range and Transmission power.

\section{A. Distance Estimation from Ranging Information}

We want to estimate Euclidean distance between node $i$ and node $j$ in Figure 1. Our algorithm assumes that there is at least one node which is in the intersection area of node $i$ and $j$ 's transmission range, and $d_{i k}$ and $d_{k j}$ are already known by the measurement with a ranging based method. Then $d_{i j}$ can be represented as follows,

$$
d_{i j}=u(z)=\sqrt{d_{i k}^{2}+d_{k j}^{2}+2 \cdot d_{i k} \cdot d_{k j} \cdot \cos (z)}
$$

However, we cannot obtain exact $\angle z$ since what we know is that node $j$ is out of node $i$ 's carrier sensing range, and node $j$ is away from node $k$ as $d_{k j}$. i.e., node $j$ exists on the blue line in Figure 1.

Theoretically $\angle z$ exists within $0 \leq z<\theta_{r}$, and $\theta_{r}$ means the largest angle of available $\mathrm{z}$ when $d_{i k}$ and $d_{k j}$ is fixed. $\theta_{r}$ is given as follows.

$$
\theta_{r}=\cos ^{-1}\left(\frac{R_{c s}^{2}-d_{i k}^{2}-d_{k j}^{2}}{2 \cdot d_{i k} \cdot d_{k j}}\right)
$$

Without $\angle z$ we are not able to compute precise $d_{i j}$, but we know available range of $z$. Using this we are going to estimate $d_{i j}$, call it $\tilde{d_{i j}} \cdot \tilde{d_{i j}}$ is the solution satisfying an equation below.

$$
\text { Minimize } e(z)=\int_{0}^{\theta_{r}}\left(d_{i j}-\tilde{d_{i j}}\right)^{2} f_{z}(z) d z
$$

where $f_{z}(z)$ represents the probability density function of $z$. In our work, we assume that nodes are uniformly distributed. It means the probability of neighbor node existence rises on increasing the distance. So we can formulate the $f_{z}(z)$,

$$
f_{z}(z)=\frac{6 \cdot u(z)}{\theta_{r}\left(u(0)+4 u\left(\frac{\theta_{r}}{2}\right)+u\left(\theta_{r}\right)\right)}
$$

Then we can compute (3), and approximate it using Simpson's rule.

$$
\tilde{d_{i j}}=\frac{6 \cdot u^{2}\left(\frac{\sin \theta_{r}}{\theta_{r}}\right)}{u(0)+4 u\left(\frac{\theta_{r}}{2}\right)+u\left(\theta_{r}\right)}
$$

\section{B. Computation of $\theta_{c}$ with Connectivity Information}

According to $d_{i k}$ and $d_{j k}, \theta_{r}$ can have a wide range. If it is possible to determine more accurate range of $z$, it will make less error in the distance estimation.

In this subsection, we define $\theta_{c}$ which is computed by using connectivity information. Connectivity information means that how many neighbor nodes are shared between two nodes. Intuitively, we can infer that the two nodes having large number of common 1-hop neighbor nodes might be close in the uniformly distributed networks. In Figure 2, the nodes in the area $S$ are the overlapped nodes between node $i$ and $j$. The number of overlapped nodes is stochastically proportional to the intersection area. Thus the intersection area is estimated by exploiting the ratio of overlapped 1-hop neighbor to the number of total 1-hop neighbor. So the ratio can be substituted for the intersection area in the geometrical formulation like below.

According to the geometries, intersection area $(S)$ in Figure 2 is,

$$
\begin{aligned}
S & =2 R_{c s}^{2} \phi-2 R_{c s}^{2} \cos \phi \sin \phi \\
& =R_{c s}^{2}(2 \phi-\sin 2 \phi)
\end{aligned}
$$

Applying the third order Taylor series expansions to Equation (7),

$$
S \approx \frac{4 R_{c s}^{2}}{3} \phi^{3}
$$

Next, we define a parameter $\rho$ which denotes a ratio of the intersection area over carrier sensing area,

$$
\rho=\frac{S}{\pi \cdot R_{c s}^{2}} \approx \frac{4}{3 \pi} \phi^{3}
$$


also it can be estimated with following equation.

$$
\tilde{\rho}=\frac{\text { Number of overlapped one hop neighbors }}{\text { Average number of one hop neighbors }}
$$

since each number of neighbor nodes and overlapped nodes is mutually independent random variables as pointed out in [4]. By substituting (10) into (9), we can obtain

$$
\theta_{c}=(6 \pi \tilde{\rho})^{\frac{1}{3}}, \quad \text { since } \theta_{c}=2 \phi
$$

\section{Range and Connectivity Combined Scheme}

As far as here, we calculate two different upper bounds of $\angle \mathbf{z}, \theta_{r}$ and $\theta_{c}$ from ranging information and connectivity information respectively, to estimate the distance using (5).

When the calculated $\theta_{r}$ has large values,

If $d_{i j}$ and $d_{k j}$,for instance, are $R_{c s}$ as maximum transmission range and $d_{i j}$ is $2 R_{c s}$, there is no intersect area and also no overlapped node. In this case, $\theta_{r}$ is $\frac{2}{3} \pi$ but it is far from true value of $\angle z$, however the result of $\theta_{c}$ is 0 and it is same as true $z$. As a instance, when the calculated $\theta_{r}$ has a large value, $\theta_{c}$ can complement the ambiguous $\angle z$. Therefore we select the definitive $\theta$ between $\theta_{r}$ and $\theta_{c}$ like below.

$$
\theta= \begin{cases}\theta_{c} & \text { if } \theta_{c}<\theta_{r} \\ \theta_{r} & \text { otherwise }\end{cases}
$$

After selecting $\theta$, the result of (5) is the estimated distance between the two-hop neighbor node.

\section{Simulation StUdies}

We conduct simulations in Matlab. In order to evaluate the proposed method, we select the HCNP [6] algorithms which uses connectivity based approach to compare with, and carry out 100 independent simulation studies in each scenario and take the average of all simulation results.

\section{A. Simulation settings}

Simulation area is a circle which has large radius of $2 \mathrm{~km}$ to remove the boundary effect. We assume node $i$ is located at the center of the simulation field. The carrier sensing range $\left(R_{C S}\right)$ is set to $300 \mathrm{~m}$. In the simulations, we select the widely used path loss model with shadow fading [7] [8].

$$
\begin{aligned}
& \operatorname{PathLoss}(i, j)=10 \cdot \alpha \cdot \log _{10} d_{i j}+\chi \\
& \text { pdf of } \chi, \quad f_{X}(x)=\frac{1}{\sqrt{2 \pi} \sigma} \cdot \exp \left(\frac{x}{2 \sigma^{2}}\right)
\end{aligned}
$$

The model is divided into a deterministic term of distance and a stochastic term that represents a shadow fading effect. For the probabilistic term, a Gaussian distribution with zero mean and standard deviation $\sigma$, is generally used. $\sigma$ is in the range of 3 to 9 depending on their surroundings [8] and in our simulations $\sigma$ takes values of $0,3,6$. Path loss exponent $(\alpha)$ have a value between 2 and 6 in accordance with an environment. In this paper, we regard that the path loss exponent is 4 . Also, we vary the number of nodes in the networks. Refer to Table 1.
TABLE I. Simulated Network DENSITY

\begin{tabular}{|c|c|c|c|}
\hline Type & Num. of nodes & Avg. density & etc \\
\hline Sparse & 60 & 4 & Urban \\
\hline Medium & 100 & 7 & Campus \\
\hline Dense & 140 & 9 & DownTown \\
\hline Very dense & 175 & 15 & Sensor Networks \\
\hline
\end{tabular}

\section{B. Simulation results}

First, we define the performance evaluation metric. Error ratio is the mean absolute distance estimation error ratio defined as

$$
\text { error ratio }=\frac{1}{N} \sum_{j=1}^{N} \frac{1}{d_{i j}}\left|d_{i j}-\tilde{d_{i j}}\right|
$$

where $j \in N_{\text {eighbor }}^{i}$, Neighbor ${ }_{2 h o p}^{i}$ means the 2hop neighbor nodes of node $i$, and $N$ is the number of Neighbor ${ }_{2 h o p}^{i}$.

The distance estimation results are plotted in Figure 3, 4, and 5. The increase of the node density brings decrease of error ratio of the HCNP in common. This is a characteristic of the connectivity based approach. However, in all simulations, our new approach is better than the HCNP. The estimation error improvement compared with the HCNP is at most 50\%, 35\%, and $18 \%$ when $\sigma$ is 0,3 , and 6 , respectively.

\section{CONCLUSION}

In this paper, we have presented a novel 2-hop neighbor distance estimation method which exploits the range and connectivity information. To improve the existing estimation accuracy, our algorithm uses the connectivity information to predict the size of the intersection area, then calculate the range of $\angle \mathrm{z}$ that overlapped nodes can exist. If this range is more specific than the result from ranging information, the algorithm adopts it. Our method can improve the estimation performance in low network density since it also uses range based approach. This is verified in previous section. Therefore, exploiting proposed algorithm makes more accurate localization, hidden terminal estimation which exists almost in two hop range, and efficient routing.

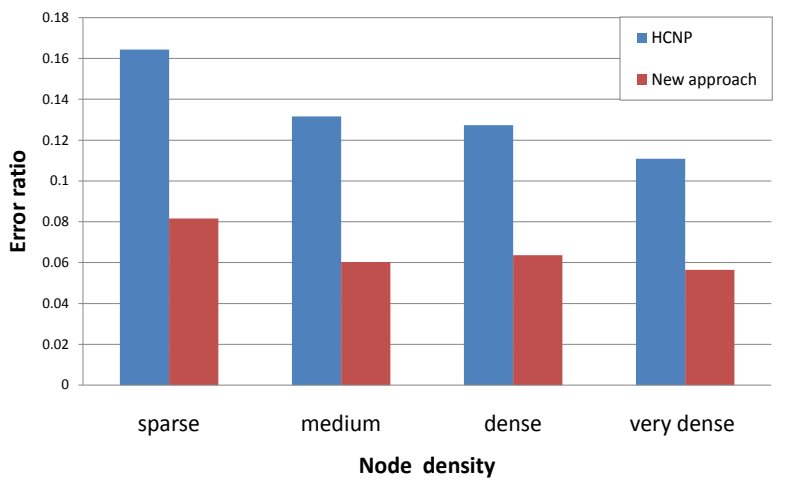

Fig. 3. Comparison of distance estimation error against the node density $(\sigma=0)$ 


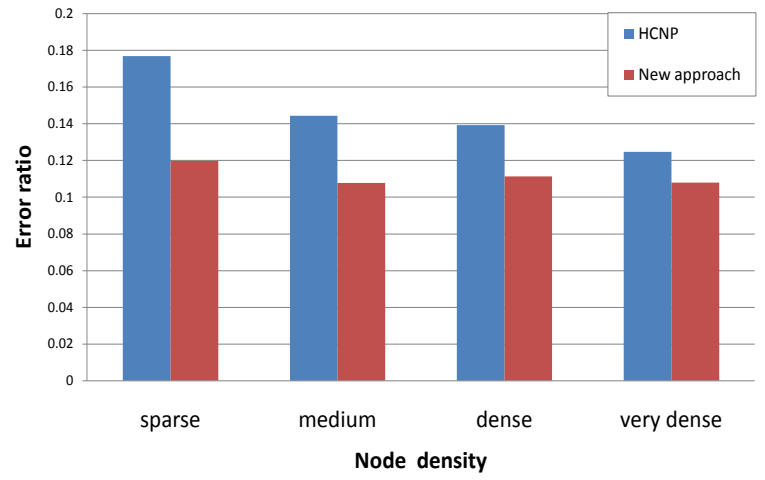

Fig. 4. Comparison of distance estimation error against the node density $(\sigma=3)$

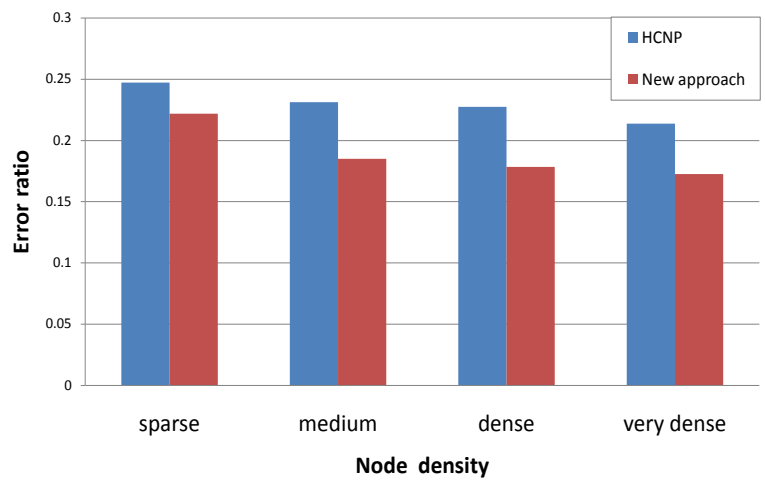

Fig. 5. Comparison of distance estimation error against the node density $(\sigma=6)$

\section{ACKNOWLEDGMENT}

This research was supported by Basic Science Research Program through the National Research Foundation of Korea( NRF) funded by the Ministry of Education, Science and Technology(NRF-2010-0008149)

\section{REFERENCES}

[1] D. Niculescu and B. Nath, "Ad hoc positioning system," Global Telecommunications Conferernce, vol. 5, pp.2926-2931, 2001.

[2] D. Ma, M. J. Er, B. Wang, and H. B. Lim, "A Novel Approach Toward Source-to-Sink Distance Estimation in Wireless Sensor Networks," IEEE Commun. Lett., vol. 14, no. 5, 2010.

[3] S. Capkun, M. Hamdi, and J. Hubaux, "GPS-free Positioning in Mobile Ad-hoc Networks," Proc. 34th Annual Hawaii Int',I. Conf. Sys. Sci., pp.3481-3490, 2001

[4] B. Huang, C. Yu, B. D. O. Anderson, and G. Mao, "Connectivitybased Distance Estimation in Wireless Sensor Networks," In Proc. IEEE GLOBECOM, pages 1-5, 2010.

[5] K. Xu, M. Gerla, and S. Bae, "Hoew Effective is the IEEE 802.11 RTS/CTS Handshake in Ad Hoc Networks?,'In Proc. IEEE Globcom, 2002

[6] D. Ma, B. Wang, H. B. Lim, and M. J. Er, "Hop-Count based Nodeto-Anchor Distance Estimation in Wireless Sensor Networks," In Proc IEEE Consumer Communications and Networking Conference, 2009

[7] C. Bettstetter, "Connectivity of Wireless Multihop Networks in a Shadow Fading Environment," IEEE Wireless Networks, vol.9, No.11, May 2005, pp.571-579

[8] P. Stuedi, O. Chinellato, and G. Alonso, "Connectivity in the presence of Shadowing in 802.11 Ad Hoc Networks,"IEEE Wireless Communications and Networking Conference Oct. 2005.

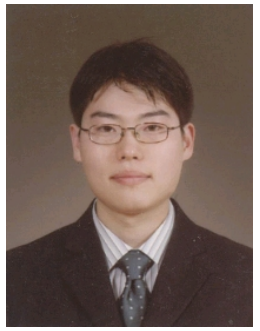

Sungchang Choi was born in Busan, Korea, on October 8, 1982. He received the B.Sc. degree in Electronics Engineering from Kumoh national institute of Technology in 2006 and M.Sc. degree in information and communization engineering from Korea Advanced Institute of Science and Technology in 2009. He is currently working toward the $\mathrm{PhD}$ degree in the Department of Electrical Engineering , Korea Advanced Institute of Science and Technology. His research interests include wireless mesh networks, MAC, routing, and localization.

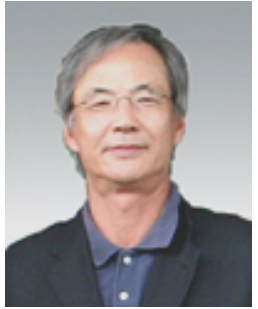

nitive networks.
Joongsoo Ma received B.Eng in Electrical Engineering, Yensei University, Seoul, Korea, 1973, and M.S and $\mathrm{Ph} . \mathrm{D}$ degrees in Electric and Computer Engineeing from University of Massachusette in 1978. He was with IBM T.J. Watson Research Center for 13 years from 1979, and was a director in SK Telecom Research Center. He is currently a professor in Korea Advanced Institute of Science and Technology. His research interests include communications network architecture and protocol, mobile communications network design, wireless mesh networks and cog- 